H. Caracciolo, S. Talay, F. Maloberti: "Optimum Selection of Capacitive Array for Multibit Sigma-Delta Modulators without DEM"; Proc. of the 16th IEEE Int. Conf. on Electronics, Circuits and Systems, ICECS 2009, Hammamet, 13-16 December 2009, pp. 759-762.

(C)20xx IEEE. Personal use of this material is permitted. However, permission to reprint/republish this material for advertising or promotional purposes or for creating new collective works for resale or redistribution to servers or lists, or to reuse any copyrighted component of this work in other works must be obtained from the IEEE. 


\title{
Optimum Selection of Capacitive Array for Multibit Sigma-Delta Modulators without DEM
}

\author{
Hervé Caracciolo ${ }^{1}$, Selçuk Talay ${ }^{1,2}$, and Franco Maloberti ${ }^{1}$ \\ ${ }^{1}$ Department of Electronics, University of Pavia, Via Ferrata, 1 - 27100 Pavia - ITALY \\ ${ }^{2}$ Electrical Engineering, Yale University, 10 Hillhouse Avenue, Dunham Laboratory, DL 501 New Haven, CT 06511
}

\begin{abstract}
A method for a smart selection and sequencing of unity capacitors in a multibit DAC is proposed. The approach, suitable for the DAC nonlinearity correction in Sigma-Delta modulators, obtains results that are better than the Dynamic Element Matching. Key of the technique is an off-line selfmeasurement of mismatches. The results significantly improve when the DAC capacitors are selected from a set that is larger than required. An affordable silicon area overhead avoids extra power consumption during the normal operation of the converter.
\end{abstract}

\section{INTRODUCTION}

Recent commercial applications in the telecommunication and sensor area utilize analog-to-digital converters (ADC) to ensure lower power and better processing. However, the challenges placed by those applications to ADC's push the ADC's to their performance limits, asking for new methods that obtain high performance but reduced power consumption. Recent applications aim at ADC's with medium-to-high resolution and very high operation speed. Since the use of digital technologies is another common constraint, the use of multi-bit Sigma-Delta $(\Sigma \Delta)$ ADC's [1] is a good choice. However, the linearity of the DAC in the ADC's is a general limitation.

There are many design dimensions that increase resolution of $\Sigma \Delta$-ADC's. Among them the order of the modulator and the oversampling ratio, normally pushed to the limits for best performance. Also increasing the number of bit of the DAC increases resolution but, as mentioned, the linearity of the multibit DAC becomes a critical design parameter. Calibration and trimming methods were introduced for multi-bit solutions. However, these methods cost overhead to the system during conversion thus, increasing the power consumption. In addition, some of the methods use blind averaging which prevents from achieving the best solution.

One well known approach is the Dynamic Elements Matching (DEM) [2] that scrambles or sequentially uses the unity elements of the DAC that, according to a given algorithm, eventually obtains noise shaping of the mismatch error. However, error is not reduced but it is only transformed into a pseudo-noise that is a possible source of tones in the signal band. Moreover, the required logic circuitry operates at the oversampled frequency, increases the consumed power and reduces the effectiveness of the converter.

This work exploit the possibility offered by the $\Sigma \Delta$ modulator to perform a preliminary measure of the value of unity elements used in the DAC. Our method uses the information for a smart selection of elements, thus avoiding DEM, while obtaining excellent performances. In the following sections the proposed approach is described. Section III the selection method of the capacitors is presented. The effect of increasing the capacitor number presented in the next section with in the simulation results. The last section concludes the paper.

\section{PRoposed METHOD}

The DAC used in $\Sigma \Delta$ modulator is made by a set of $N$ unity capacitors, $C_{u}$, nominally equal. The mismatch among them makes each

$$
C_{i}=C_{u}+\varepsilon_{i}
$$

The sequence of errors $\varepsilon_{i}$ has a systematic and random component that cause a deviation of the transfer characteristic of the DAC from the linear behavior. The DEM technique transform the error to into a pseudo-noise whose input referred voltage power is

$$
V_{n, \varepsilon}^{2}=\frac{V_{R}^{2}}{C_{u^{2}}} \sum_{i=1}^{N} \varepsilon_{i}^{2} .
$$

A random scrambling spreads that power uniformly over the Nyquist interval whereas other methods may obtain shaping. The result is normally suitable for a $\sigma$ of mismatch well below $1 \%$ and resolution of $12-14$ bit.

Suppose now to know the value of the sequence of errors, $\varepsilon_{i}$. Instead of dynamic use of elements we can consider a "smart" use of elements that optimize the INL of the DAC. Assuming to have just a systematic error, the INL may look like the one of Figure 1 (a). A proper sequence of the unity elements can change the INL diagram into the one of Figure 1 (b) that has a lower maximum amplitude and a more busy behavior.
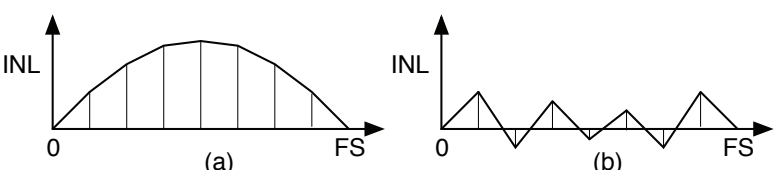

Fig. 1. INL of a) a systematical error b) a proper sequence

Notice that the INL of Fig. 1 (b) is not a good choice for small input amplitudes in a Nyquist rate DAC but is optimal 


\begin{tabular}{|c|c|c|c|c|c|}
\hline & $\begin{array}{c}\text { not } \\
\text { used }\end{array}$ & $\begin{array}{c}\text { not } \\
\text { used }\end{array}$ & 4 & $\begin{array}{c}\text { not } \\
\text { used }\end{array}$ \\
\hline $\begin{array}{c}\text { Selection } \\
\text { and } \\
\text { Sequence } \\
\text { Logic }\end{array}$ & 6 & 1 & 7 & $\begin{array}{c}\text { not } \\
\text { used }\end{array}$ \\
\cline { 2 - 5 } & 3 & $\begin{array}{c}\text { not } \\
\text { used }\end{array}$ & 5 & $\begin{array}{c}\text { not } \\
\text { used }\end{array}$ \\
\hline $\begin{array}{c}\text { not } \\
\text { used }\end{array}$ & 8 & $\begin{array}{c}\text { not } \\
\text { used }\end{array}$ & 2 \\
\hline
\end{tabular}

Fig. 2. Layout diagram of a possible capacitor array

when the DAC is used in a $\Sigma \Delta$ modulator. The digital output that controls the DAC is the input plus a shaped quantization noise, whose maximum amplitude is the quantization step. Therefore, the control of the DAC is inherently a dynamic averaging of the DAC levels corresponding to the input input amplitude over two or more neighbor quantization intervals. The effect, as verified shortly by computer simulations, is to smooth the effect and to obtain negligible harmonic tones.

Suppose that the available number of elements is more than what is needed by the DAC. For example 9 or 16 when 8 are needed for a 3-bit DAC. Knowing the mismatches it is possible to choose the most favorable elements for the optimal sequence. This, as verified by simulations further improves the INL. Hence, the proposed approach provide different levels of accuracy depending on the silicon area that can be allocated for this capacitors and the control logic. For example, with a 3-bit DAC, 16 elements can be arranged in a square matrix and the smart selection can use the elements and the sequence of Fig. 2, where a suitable logic determines the capacitances to be used and the sequence.

The circuit implementation with more capacitors (i.e. 16) and 3-bit DAC can use separate elements or the same elements for the sampling of input and for the DAC. In the latter case, it is necessary to generate an extra logic signal that identifies the set of unity elements to be charged to the input, for avoiding an extra capacitive load to the input terminal. An elementary generic element and the used switches are shown in Fig. 3. It uses two controls signals one for the selection and the other for the DAC function.

\section{ERROR MEASUREMENT}

The value measurement of the $\mathrm{N}$ capacitors of the array is performed off-line using the $\Sigma \Delta$ architecture itself. The scheme is re-configured as incremental [4], [5], i.e. the charge stored in two integrators is reseted. A fixed voltage, $V_{\text {Meas }}$

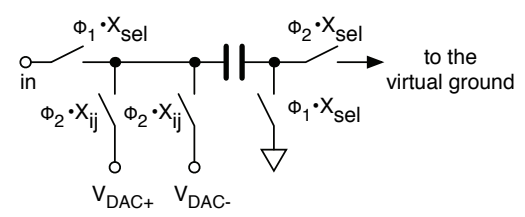

Fig. 3. Switched capacitor scheme of the single unity element. lower than $V$ ref $/ N$ is applied to the input. The output of the modulator is a bit-stream that uses none or just one capacitor only, the one under measure, $C_{i}$. The use of a cascade of integrators (one more than the order of the modulator) provides the digital measure of the input voltage multiplied by the input gain

$$
V_{\text {out }, i}=\frac{C_{i}}{C_{f}} V_{\text {Meas }}
$$

since $C_{f}$, the feedback capacitance is $\sum_{1}^{N} C_{i}$, the digital output is the measure of $C_{i}$ multiplied by an unknown ratio. If the same reference voltage is used for the measure with all the unity elements, the results are a relative measure of the values that enables to estimate the mismatches.

With a second order modulator the use of three cascades integrators for $K$ clock periods obtain $K(K+1)(K+2) / 6$ as full scale. Therefore, the accuracy of the measurement is approximately $1 /\left(3 \log _{2} K-3\right)$. Therefore by selecting $K=2^{8}$ the relative value of each capacitor is measured with more than 20 bit of accuracy.

Once the measurement for a capacitor is performed, the set with the best matching is selected. Then, a suitable algorithm for having the minimum INL is performed.

\section{OptIMUM SELECTION}

The optimum selection of $\mathrm{N}$ elements out of $\mathrm{M}$ available corresponds to the set with minimum relative differences. A possible algorithm, used in the simulations described in the next section, employs the partial average value $\bar{C}_{i}$ taken from a set of $(K-1)$ elements that excludes $C_{i}$

$$
\bar{C}_{i}=\frac{1}{K-1} \sum_{j \neq i} C_{j} .
$$

The selections starts with $K=M$ and eliminates the element for which the distance $D_{i}$

$$
D_{i}=\left(C_{i}-\bar{C}_{i}\right)^{2}
$$

is maximum. Then, $K=M-1$ and the test continues with eliminations of one by one element until reaching the number of elements required by the DAC.

The choice of the optimal sequence of $N$ elements, $C_{1}, C_{2}, \cdots, C_{N}$, whose average value is $\bar{C}=1 / N \sum C_{i}$, is done according to the following algorithm:

The first selected element, $C_{s, 1}$ is the one that obtains the minimum value of

$$
\Delta_{1}=\left(C_{i}-\bar{C}\right)^{2} .
$$

The next element obtains the minimum

$$
\Delta_{2}=\left(C_{i}+C_{s, 1}-2 \bar{C}\right)^{2},
$$

and so forth. The selected elements and the optimal sequence control a switch matrix that uses the thermometric control of the DAC to provide the logic signals used in the switched capacitor scheme of Fig. 3. Fig. 4 gives an example of possible 


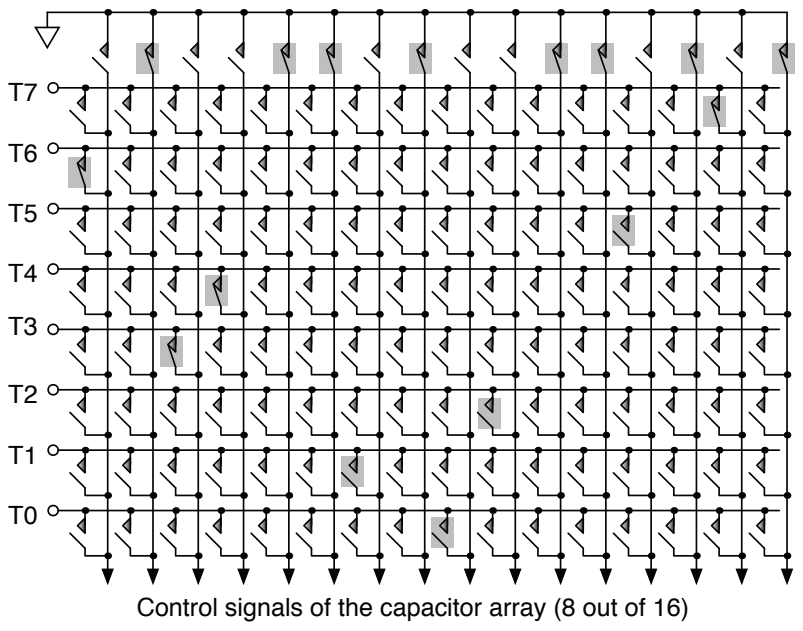

Fig. 4. Matrix of switches suitable for the control of a DAC that uses 8 out of 16 unity elements.

switch matrix with 16 unity elements for a 3-bit DAC. The measure of the elements value and the described algorithms select the first, third, fourth and so forth capacitors. The control of the others is grounded by the switches on the top. The first capacitor is used to convert the bit T6 of the thermometric signal, the third converts T3 and so forth. Therefore, the circuit overhead is the matrix of Fig. 4 and the memory that gives the static controls of switches. The handled signal is digital and the use of a switch does not affect, in practice, the speed.

\section{Simulation Results}

The proposed approach has been simulated at the behavioral level with various mismatches and signal amplitudes. The first study concerned the evaluation of the INL in different foreseen cases. Fig. 5 shows the statistical distribution of the absolute value of the INL obtained with a 3-bit DAC made by unity capacitances with random mismatch with $\sigma=0.01$. The distribution concerns 100 cases. The Value of the INL ranges from 0.7 to $2.7 \%$. The average loss for the SNR in a

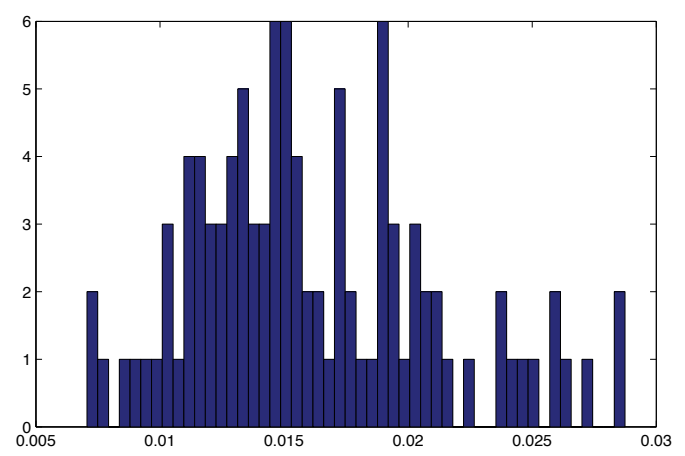

Fig. 5. Maximum absolute value of the INL of a 3-bit DAC with a $0.01 \sigma$ error.

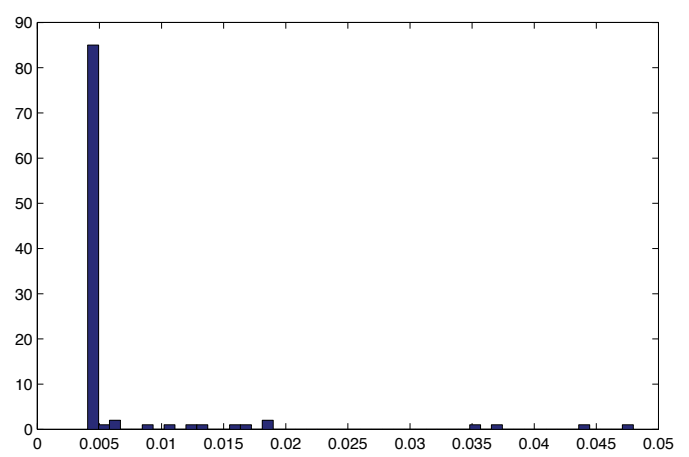

Fig. 6. Maximum absolute value of the INL of a 3-bit DAC with a $0.01 \sigma$ error, after optimum selection.

3-bit second-order modulator with $\mathrm{OSR}=32$ is $15 \mathrm{~dB}$. Moreover a tone at $-79 \mathrm{~dB}$ with $-6 d B_{F S}$ appears. Starting from same mismatched cases, with only 8 elements the algorithm of optimum sequence obtains the histogram of Fig. 6. The distribution is strongly concentrated around 0.005 that is half the value of the used $\sigma$. Moreover, the proper sequence makes the INL plot suitable for smoothing thanks to the quantization noise random variation.

The histogram of Fig. 6 shows that a small number of cases are not properly brought to a low value of INL. They are caused by the mathematical tails of the statistic distribution that foresee values of unity capacitances with a large error. Indeed, in real situations having a big error is not possible and the real statistical distribution is not with a fully random added term.

Notice that in order to obtain an INL curve like the one of Fig. 1 (b) it is necessary to have pair of capacitors whose value are symmetrical with respect to the average. If, for example, the set of 8 capacitors is made by 5 elements bigger and 3 smaller than the average value the algorithm cannot pair a bigger and a smaller element. Consequently, the INL will show, somewhere, two consecutive increases. Obviously

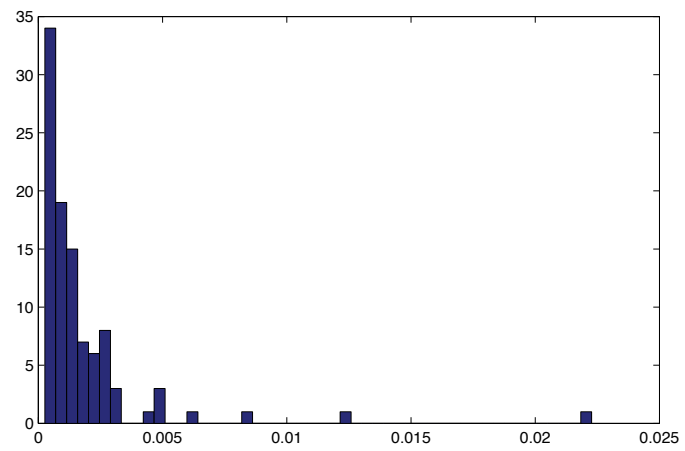

Fig. 7. Maximum absolute value of the INL of a 3-bit DAC with a $0.01 \sigma$ error with optimum selection and 9 elements. 


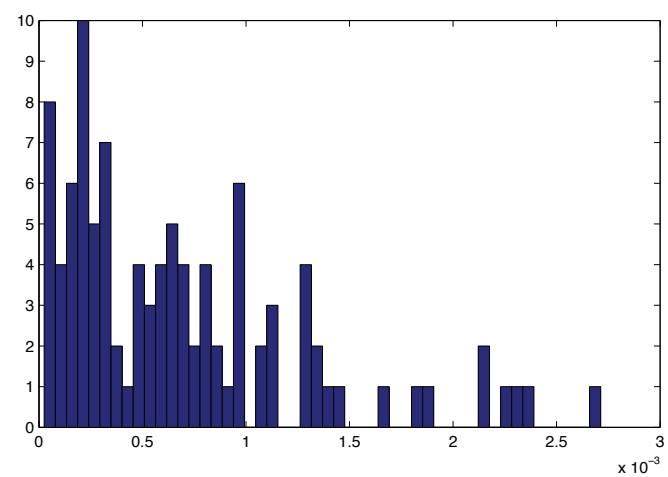

Fig. 8. Maximum absolute value of the INL of a 3-bit DAC with a $0.01 \sigma$ error with optimum selection and 16 elements.

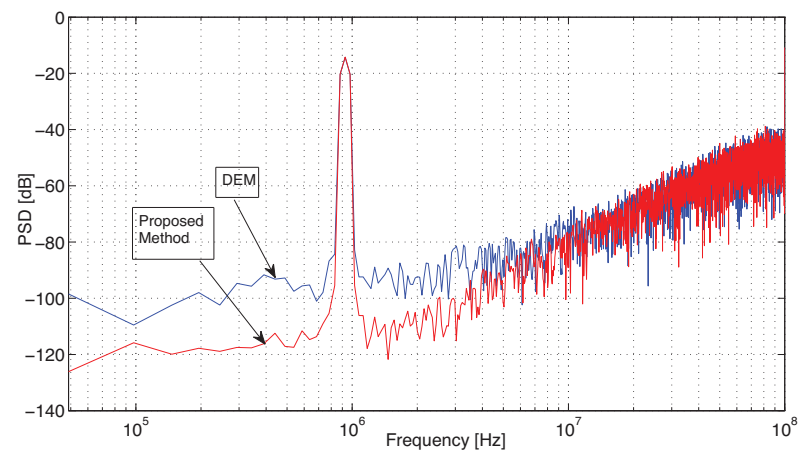

Fig. 9. Output spectra obtained with the proposed method and DWA-DEM. The capacitor mismatch in the 3-bit DAC is $\sigma=0.01$.

the situation is worst with a $6-2$ or a $7-1$ distribution around the average. These cases and the the effect of tails are the possible limit the yield even when considering the real statistical distribution.

A possible systematic error in the unity capacitor values is transformed into alternate fluctuations of the INL that, as mentioned is smoothed by the effect of the quantization error. Simulation results shows that the effect of systematic contributions is negligible until very large gradients that cause an overall change of unity elements as large as $10-15 \%$.

The result of Fig. 6 denotes a good improvement but some worst cases make the possible yield non acceptable. In order to improve the effectiveness of the method the 8 elements are selected from a set with extra parts. The statistic distribution significantly improves with only one extra capacitor, as shown in Fig. 7. The value of the INL is often below $0.2 \%$ with few cases of values larger than $0.6 \%$. The worst situations correspond to the $6-2$ distribution around the average because the probability to have $7-1$ is almost zero.

Obviously, the use of more element to perform the selection improves the INL histogram. Fig. 8 shows that with 16 elements and optimum sequence the INL improves by almost an order of magnitude with respect to the plain case.

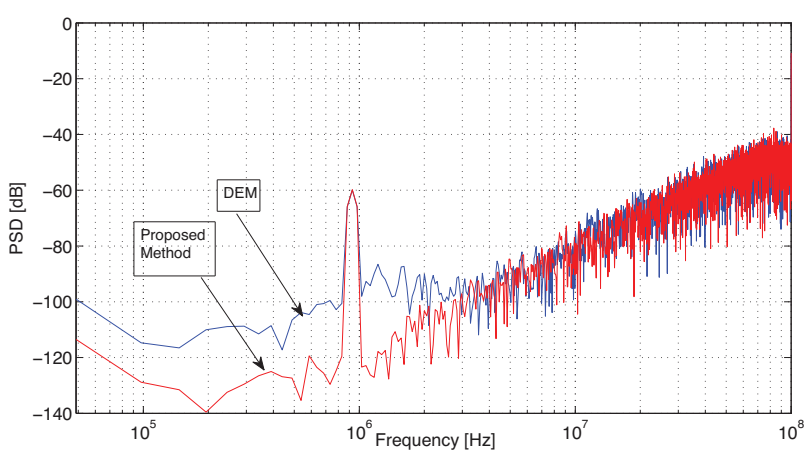

Fig. 10. Output spectra of the case of Fig. 9 with $-60 \mathrm{~dB}$ input amplitude.

The improved INL and the optimum sequence obtain performances better than the DEM with a mismatch as large as $1 \%$. Fig. 9 shows the output spectra the SNR of a second order fully differential modulator for the proposed method, that is unchanged with respect to the ideal case, and the spectrum of a modulator with DWA-DEM that starts deviating from the ideal response for OSR $>20$. The average SNR loss with OSR $=32$ is $7 \mathrm{~dB}$.

The benefit of the method is unchanged for low input amplitudes. Fig. 10 shows that the spectra (with a different set of unity capacitors and $\sigma=0.01$ ) have similar behavior. The one of the proposed method still follows the case without mismatch, thus demonstrating the smoothing effect of the quantization noise.

\section{CONCLUSION}

A linearization technique for the DAC used in Sigma-Delta modulators has been presented. The proposed method relies on the mismatch self-measurement capability of $\Sigma \Delta$ modulators and uses the mismatch data for selecting and sequencing in an optimum manner such that the capacitors used in the multi-bit DAC compensates for the mismatch error of the capacitors in multibit DAC. The method obtains better results than dynamic matching and do not requires extra power during the normal operation.

\section{ACKNOWLEDGMENT}

The authors thanks FIRB, Italian National Program, Project RBAP06L4S5, for a partial financial support.

\section{REFERENCES}

[1] S. R. Norsworthy, R. Schreier, and G. C. Temes, Eds., Delta-Sigma Data Converters. Piscataway, NJ: IEEE Press, 1997.

[2] Y. Geerts, M. Steyaert, and W. Sansen, Design of multi-bit Delta-Sigma $A / D$ converters. Boston: Kluwer Academic Publishers, 2002.

[3] C. Wegener, and M.P. Kennedy, "Linear model-based error identification and calibration for data converters," Design, Automation and Test in Europe Conference and Exhibition (DATE), 2003, pp. 630-635

[4] S. Kavusi and H. Kakavand, and A. El Gamal, "On Incremental SigmaDelta Modulation With Optimal Filtering, IEEE Transaction on Circuits and Systems I, TCAS-I, pp. 1004-1015, May 2006.

[5] A. Agnes and F. Maloberti, "Multi-bit High-Order Incremental Converters with Digital Calibration', Proc. of European Conference on Circuit Theory and Design (ECCTD), August 2009. 